\title{
ANÁLISE QUANTITATIVA DAS ATITUDES DE ESTUDANTES DE ENGENHARIA NO PROCESSO DE APRENDIZAGEM MATEMÁTICA
}

\author{
Anna Regina Corbo - anna.costa@ cefet-rj.br \\ Departamento Acadêmico de Matemática - DEMAT - CEFET/RJ \\ Av. Maracanã, 229 - Maracanã \\ 20271-110 - Rio de Janeiro - RJ
}

Alexandre Esteves Ribeiro - xandeae2016@gmail.com

Graduando em Engenharia de Produção - CEFET/RJ

\author{
Maria Clara Palermo de Souza Carvalho Caria - mariaclarapll@gmail.com \\ Bolsista PIBIC - CEFET/RJ \\ Graduanda em Engenharia de Produção - CEFET/RJ
}

\begin{abstract}
Resumo: A percepção do aluno sobre uma disciplina pode ser um dos fatores responsáveis por oferecê-lo a motivação necessária para seu aprendizado. Este trabalho investiga a relação existente entre a matemática e o estudante de engenharia, com o objetivo de mensurar a importância dessa relação na formação acadêmica e pessoal do aluno. Para isto, utilizou-se como instrumento o MAPS (Mathematics Attitudes and Perceptions Survey), um formulário padronizado com 32 afirmações, desenvolvido na University of British Columbia (EUA) em 2016. O MAPS visa perceber a existência de uma relação entre aprendizagem e afinidade de um aluno com a matemática. Estas afirmações são separadas em categorias que tratam de habilidades matemáticas relacionadas à confiança, solução de problemas, maturidade, interesse, vida real, coerência, entre outras. Desta forma, pode-se verificar em quais áreas os alunos têm suas perspectivas mais próximas ou afastadas de um padrão favorável à aprendizagem pré-estabelecido por especialistas. O levantamento foi aplicado em um grupo de 104 alunos de segundo e terceiro anos de várias habilitações de engenharia em uma instituição pública brasileira. Os resultados foram tratados estatisticamente de modo a correlacionar as respostas obtidas com o padrão esperado para o bom ambiente de aprendizagem matemática. A aplicação do MAPS apresentou dados pertinentes que permitiu avaliar como um todo a existência de um vínculo entre o desempenho do aluno e de sua relação de afinidade com a matemática.
\end{abstract}

Palavras-chave: Aprendizagem matemática. Engenharia. Diagnóstico de atitudes. MAPS.

\section{INTRODUÇÃO}

O sucesso de um aluno em um processo de aprendizagem matemática muitas vezes é descrito como a capacidade desse aluno em resolver problemas. A resolução de problemas é essencial para o aprendizado da matemática, pois ajuda o aluno a desenvolver seu processo cognitivo e o torna mais receptivo para a resolução de problemas mais complexos (SADIYAH et al., 2019). É cada vez mais crescente o movimento de formular estratégias para reduzir as dificuldades apresentadas pelos alunos em resolver problemas como novos métodos de ensino ou novos materiais de ensino.

No entanto, também é importante considerar a atitude dos alunos em relação à matemática. Várias pesquisas apontam a importância das atitudes e disposição dos alunos para aprender matemática (ALVES et al., 2016; MARMITT et al., 2015; ROCHA, 2103). 
Defende-se a ideia de que a questão do comprometimento dos alunos com relação a sua aprendizagem está impregnada de diferentes fatores dentre eles os citados por Lester (1980): interesse, motivação, confiança e perseverança que são componentes do domínio afetivo.

No caso particular da engenharia, a matemática desempenha um papel importante, uma vez que está ligada a diversas áreas primordiais da vida quotidiana. Vários aspectos da atividade de engenharia compreendem a formulação de problemas e a escolha de métodos adequados para resolvê-los. Independentemente da área, os conceitos matemáticos são essenciais na formação de engenheiros, seja na compreensão de diferentes conceitos, seja no conhecimento específico da sua aplicabilidade. Durante o curso, os estudantes aprendem e consolidam os princípios básicos de matemática para resolver problemas práticos, reforçando o conhecimento de conceitos matemáticos, em áreas como estatística, métodos numéricos, otimização e simulação, dentre outros.

Apesar da matemática constituir uma disciplina-base na admissão a cursos de engenharia, são identificadas inúmeras dificuldades por parte dos estudantes desses cursos. Vários estudos evidenciam as dificuldades sentidas pelos estudantes na transferência dos conhecimentos matemáticos para o contexto da Engenharia (ver, por exemplo: FADALI et al., 2004; GYNNILD et al., 2005). Da experiência de muitos professores do ciclo básico resulta o reconhecimento de que as notas dos estudantes revelam dificuldades e questões motivacionais que poderão ir muito além do conhecimento matemático necessário.

Diversos pesquisadores têm se dedicado a analisar as atitudes, principalmente dos professores, e seus impactos no processo de aprendizagem matemática. No entanto, são mais raras as investigações com estudantes em especial com alunos de graduação. Dentre os estudos quantitativos existentes podemos citar o realizado com alunos de Administração em Gottschall \& Garcia-Bayonas (2008), com alunos de Biologia em Flanagan \& Einarson (2017) e com alunos de Engenharia por Alves et al. (2016).

\subsection{Mathematics Attitudes and Perceptions Survey (MAPS)}

O levantamento MAPS (do inglês, Mathematics Attitudes and Perceptions Survey) desenvolvido por Code et al. (2016) na University of British Columbia com enfoque no aprendizado de matemática de alunos de graduação em bacharelados relacionados à STEM (science, technology, engineering and mathematics). Inicialmente o MAPS foi projetado com base no formulário CLASS (Colorado Learning Attitudes about Science Surveys) desenvolvido na Universidade do Colorado por Adams et al. (2006) e aplicado, inicialmente, ao campo da Física, visando perceber a existência de uma relação entre aprendizagem e afinidade de um aluno com a ciência em geral. O objetivo principal do MAPS é analisar quantitativamente a relação emocional dos alunos com a matemática.

O levantamento consiste em uma sequência de afirmações separadas em categorias que tratam de habilidades matemáticas relacionadas à confiança, solução de problemas, maturidade, interesse, relações da matemática com o mundo real, coerência e natureza das respostas. Desta forma, pode-se verificar em quais áreas os alunos têm suas perspectivas mais próximas ou afastadas de um padrão favorável à aprendizagem pré-estabelecido por especialistas.

O formulário é padronizado com 32 afirmações. Estas afirmações foram aleatoriamente apresentadas aos participantes do estudo de modo que não fosse possível, durante a coleta de dados, observar um padrão de intenção. Das 32 afirmações, 30 se encaixam em uma das sete categorias citadas na Figura 1, uma não possui categoria e a afirmação restante (sentença número 19) é uma afirmação filtro para controle dos que efetivamente participaram da pesquisa e sem importância teórica para o estudo. As afirmações foram organizadas segundo a 
Tabela 1 e o conteúdo de cada uma das afirmações foi livremente traduzido de Code et al (2016) pelos autores.

Figura 1 - Esquema de categorias analisadas pelo levantamento MAPS.

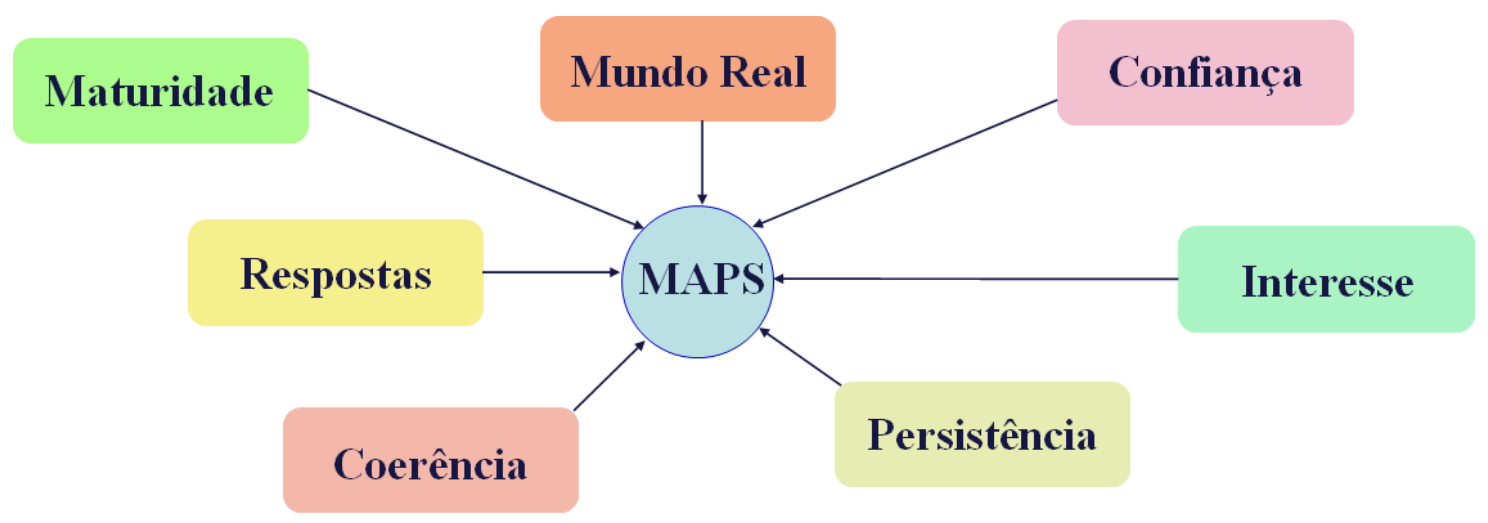

Fonte: autores (2020)

O formulário é padronizado com 32 afirmações. Estas afirmações foram aleatoriamente apresentadas aos participantes do estudo de modo que não fosse possível, durante a coleta de dados, observar um padrão de intenção. Das 32 afirmações, 30 se encaixam em uma das sete categorias citadas na Figura 1, uma não possui categoria e a afirmação restante (sentença número 19) é uma afirmação filtro para controle dos que efetivamente participaram da pesquisa e sem importância teórica para o estudo. As afirmações foram organizadas segundo a Tabela 1 e o conteúdo de cada uma das afirmações foi livremente traduzido de Code et al (2016) pelos autores.

Tabela 1 - Organização das afirmações nas categorias do estudo.

\begin{tabular}{|l|l|}
\hline Categoria & Número das afirmações \\
\hline Maturidade & $5,6,22,31$ \\
\hline Relações com o mundo real & $13,15,21,25$ \\
\hline Confiança & $1,14,17,20$ \\
\hline Interesse & $12,26,32$ \\
\hline Persistência & $8,10,24,29$ \\
\hline Coerência & $3,4,11,18,23$ \\
\hline Natureza das respostas & $2,7,9,16,28,30$ \\
\hline Sem categoria & 27 \\
\hline
\end{tabular}

Fonte: Adaptado de Code et al (2016)

As afirmações e a resposta oficial foram construídas com base em um consenso da opinião de vários professores especialistas por processo descrito em Code et al (2016). As opções de resposta seguem a escala Likert, variando entre 1 - Discordo Fortemente, 2 Discordo, 3 - Neutro, 4 - Concordo e 5 - Concordo Fortemente. Para a verificação das amostras coletadas estabeleceu-se que, caso o padrão oficial indicasse a opção (2 - Discordo), as respostas (1 - Discordo Fortemente) e (2- Discordo) dos alunos seriam consideradas concordantes com o padrão. Do mesmo modo, caso o padrão oficial indicasse (4- Concordo), 
"Os desafios para formar hoje o engenheiro do amanhã"

as respostas (4-Concordo) e (5-Concordo Fortemente) seriam consideradas concordantes com o padrão.

\section{MATERIAS E MÉTODOS}

Este estudo foi desenvolvido com alunos de graduação em engenharia do Centro Federal de Educação Tecnológica Celso Suckow da Fonseca (CEFET-RJ), no Rio de Janeiro, durante o ano de 2019. Os cursos de engenharia da instituição seguem as seguintes ênfases: Engenharia Ambiental, Automação, Civil, Elétrica, Eletrônica, Mecânica, Produção e Telecomunicações.

Para a realização do estudo foi aplicado a um grupo de alunos o levantamento MAPS descrito na seção 1.2. O formulário era identificado e foi aplicado de modo online, ao fim do primeiro e do segundo semestre de 2019. As afirmações foram agrupadas de acordo com o que se desejava avaliar dentre as categorias citadas na seção 1.2 .

Foram coletados 114 formulários nos dois semestres de 2019. No entanto, 10 formulários foram descartados por motivos diversos (não atendiam ao perfil desejado, haviam marcado erroneamente a questão filtro e/ou outros motivos), totalizando 104 amostras para a análise. $\mathrm{O}$ perfil dos alunos participantes consistia em alunos que estivessem, no mínimo, no terceiro semestre e já aprovados em Cálculo I. Deste modo, suponha-se por parte dos entrevistados um certo grau de maturidade.

Os alunos classificaram privadamente as afirmações e os dados coletados foram explorados posteriormente. Analisou-se o somatório das respostas que seguiam o padrão prédeterminado, gerando um percentual de concordância por categoria.

\section{RESULTADOS E DISCUSSÃO}

Existem muitas pesquisas investigando as atitudes, as concepções e as crenças dos estudantes em relação à matemática. Os dados variam de estudo para estudo, dependendo das variáveis analisadas. Segundo Marmitt et al. (2015), o tipo de escola, as questões culturais dos países, o grau de ensino investigado, a idade dos estudantes, por exemplo, pode contribuir para as divergências encontradas. O objetivo de nossa pesquisa é mensurar como as categorias de cunho socioemocional aqui listadas impactam nas atitudes dos alunos da instituição durante o processo de aprendizagem de matemática. Para tal, analisaremos o resultado global e os resultados por categoria, elegendo uma afirmação representativa por assunto para exibir e discutir os resultados obtidos.

Nesta pesquisa, foram analisados 104 formulários respondidos voluntariamente por alunos de graduação de todos os cursos de engenharia da instituição. Na amostra, 26,9\% dos entrevistados eram do sexo feminino e $73,1 \%$ eram do sexo masculino.

A partir desta amostra é possível observar no Gráfico 1 que, de modo geral, os alunos apresentaram bons níveis de consonância com o padrão oficial nas categorias Maturidade, Relações com mundo real e Interesse (todos acima de $70 \%$ de correspondência). Por outro lado, as categorias Confiança e Natureza das respostas apresentaram desempenho mediano (52,4\% e $51,28 \%$ de consonância respectivamente). Além disso, detectou-se um baixo nível de Persistência com somente 46,39\% de alinhamento com o padrão, entre os entrevistados.

\subsection{Maturidade}

Esta categoria classifica a crença dos alunos sobre se a capacidade matemática é inata ou pode ser desenvolvida. Tomamos aqui a seguinte afirmação representativa para a categoria maturidade: A habilidade matemática é algo que nasce com a pessoa e não pode ser 


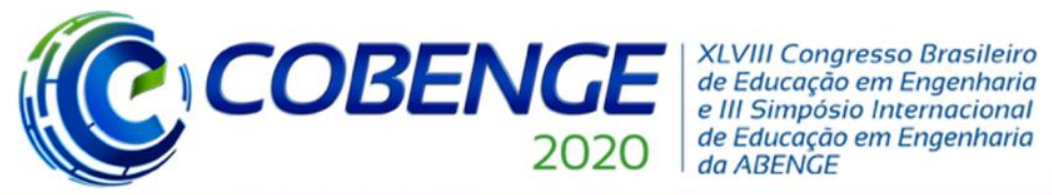

"Os desafios para formar hoje o engenheiro do amanhã"
$\mathrm{Ol} \mathrm{a} \mathrm{O3}$ de dezembro Evento On-line

adquirida. No Gráfico 2 é possível notar que, a grande maioria dos alunos discordaram da afirmação, com $87,5 \%$ dos entrevistados, ao passo que somente $4,8 \%$ do total de alunos foram favoráveis à afirmação.

Gráfico 1 - Percentual de respostas consoantes com o padrão, por categoria.

$100,00 \%$

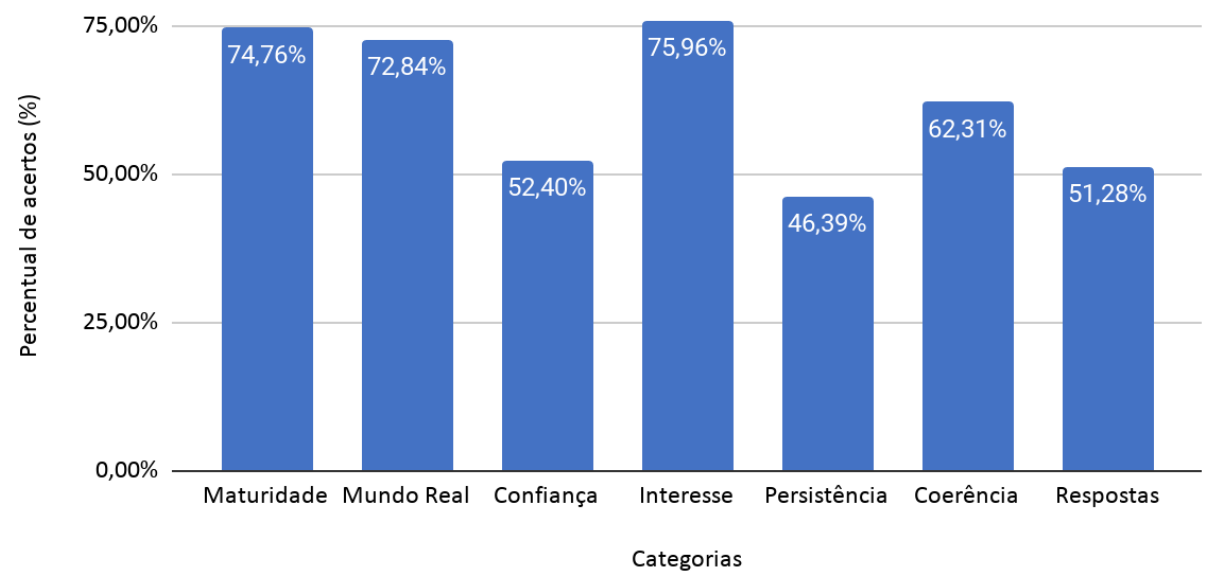

Gráfico 2 - Resultados para a afirmação representativa da categoria Maturidade.

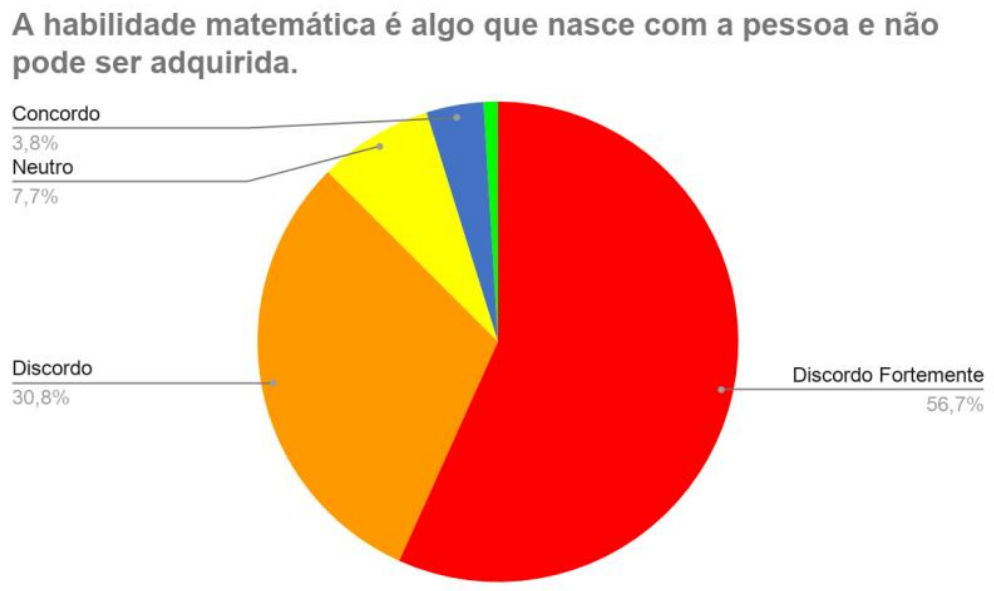

\subsection{Relações entre a matemática e o mundo real}

A categoria visa correlacionar as relações percebidas pelos alunos entre a matemática estudada e o mundo real. Para representar esta categoria, analisaremos a seguinte afirmação: $A$ matemática escolar tem pouco a ver com o que eu vivencio no mundo real.

No Gráfico 3 observa-se que, $47,1 \%$ do total dos entrevistados discordaram da afirmação e, por outro lado, o percentual de alunos que concordaram foi de $27,9 \%$. 


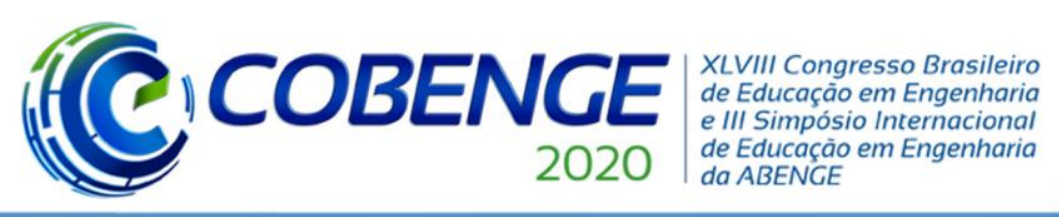

"Os desafios para formar hoje o engenheiro do amanhã"

Gráfico 3 - Resultados para a afirmação representativa da categoria Mundo Real.

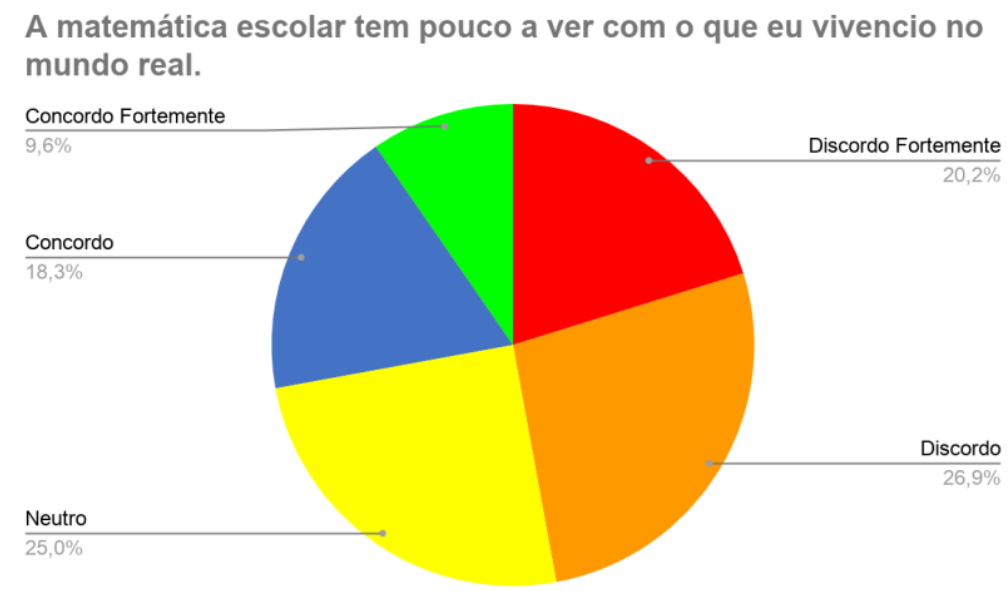

\subsection{Confiança}

A confiança aqui analisada é a capacidade percebida por uma pessoa de se envolver com sucesso em tarefas matemáticas. A confiança pode afetar a disposição do aluno de se envolver com uma tarefa, no esforço que eles gastam no trabalho e o grau em que persistem ao encontrar contratempos. Neste trabalho tomamos a seguinte afirmação representativa para a categoria confiança: Não importa o quanto eu me prepare, eu ainda não fico confiante quando faço provas de matemática.

É possível observar no Gráfico 4 que, no total, 39,4\% dos entrevistados discordaram de tal afirmação enquanto $33,7 \%$ dos entrevistados concordaram. Além disso, foi representativo o índice de neutralidade entre os alunos onde $26,9 \%$ dos entrevistados não concordaram nem discordaram da afirmação.

Gráfico 4 - Resultados para a afirmação representativa da categoria Confiança.

Não importa o quanto eu me prepare, eu ainda não fico confiante quando faço provas de matemática.

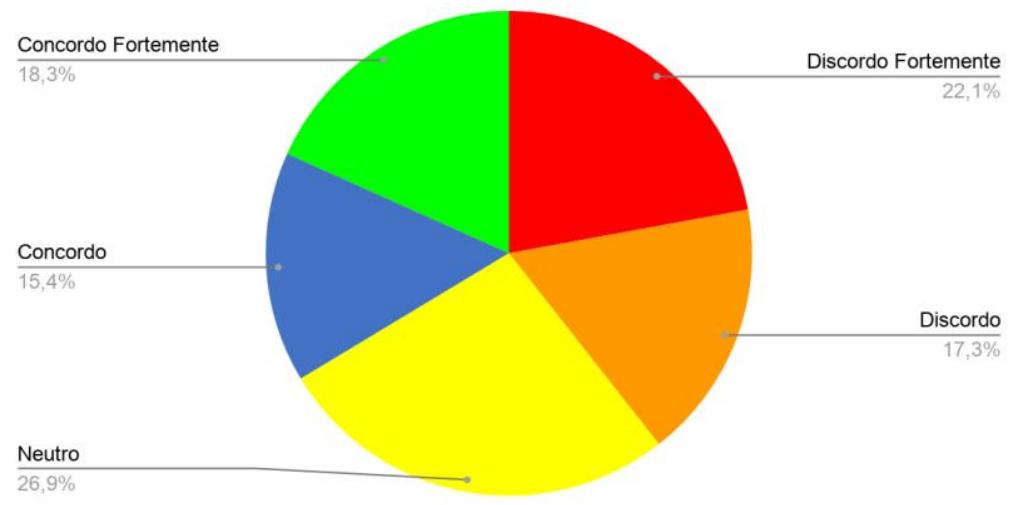

\subsection{Interesse}

Esta categoria quantifica o interesse dos alunos em se envolver com a matemática. A seguinte afirmação foi representativa para a categoria interesse: Só aprendo matemática quando me é requisitado.

De acordo com o Gráfico 5 observa-se que uma grande parcela dos entrevistados discordou da afirmação, no total de $62,5 \%$, contudo apenas $12,5 \%$ dos entrevistados concordaram com a afirmação. 


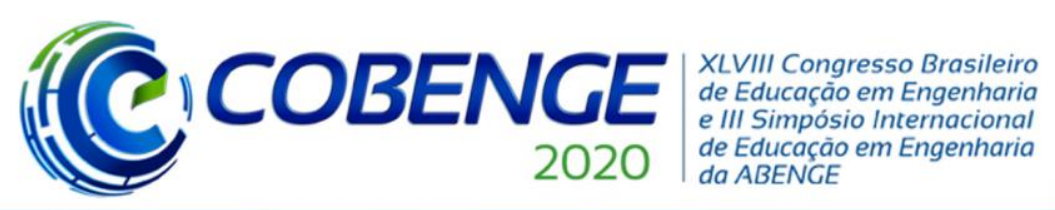

"Os desafios para formar hoje o engenheiro do amanhã"

Gráfico 5 - Resultados para a afirmação representativa da categoria Interesse.

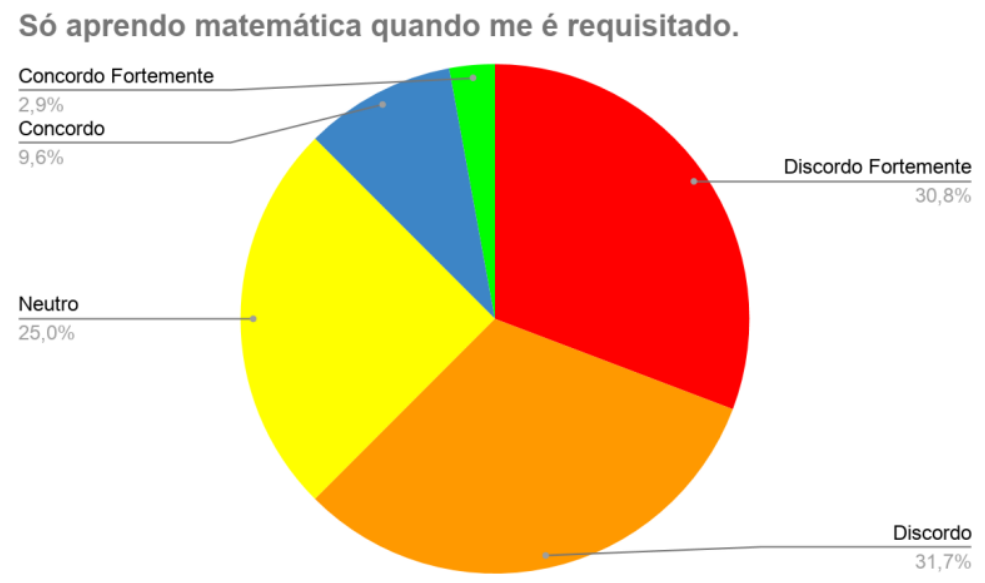

\subsection{Persistência}

O modo como os alunos abordam a solução de um problema matemático não trivial (isto é, onde eles podem "ficar presos") é tão importante quanto sua capacidade de resolver esse problema. Para analisar esta característica, tomamos a seguinte afirmação representativa para a categoria persistência: Eu fico chateado facilmente quando travo em uma questão de matemática.

No Gráfico 6 é possível notar que, o percentual de alunos que se posicionaram concordando com a sentença foi de $64,4 \%$, já o percentual de participantes que discordaram foi de somente $17,3 \%$.

Gráfico 6 - Resultados para a afirmação representativa da categoria Persistência.

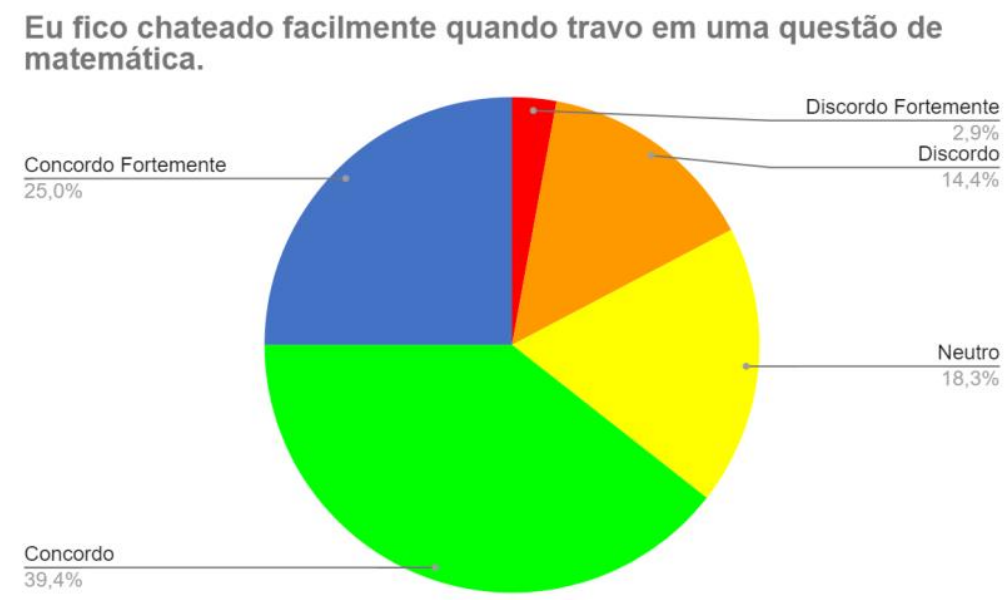

\subsection{Coerência}

Esta categoria visa quantificar as perspectivas dos alunos sobre a natureza de seus conhecimentos matemáticos pessoais. Para exemplificar o analisado nesta categoria, tomamos a seguinte afirmação representativa: Em matemática, eu acho importante que as fórmulas e os procedimentos façam sentido antes de usá-los.

De acordo com o Gráfico 7 é possível observar que, de um modo geral os entrevistados se posicionaram em sua grande maioria a favor da afirmação, atingindo um percentual de 78,8\%, porém somente $7,7 \%$ do total de alunos discordaram da sentença. 
Gráfico 7 - Resultados para a afirmação representativa da categoria Coerência.

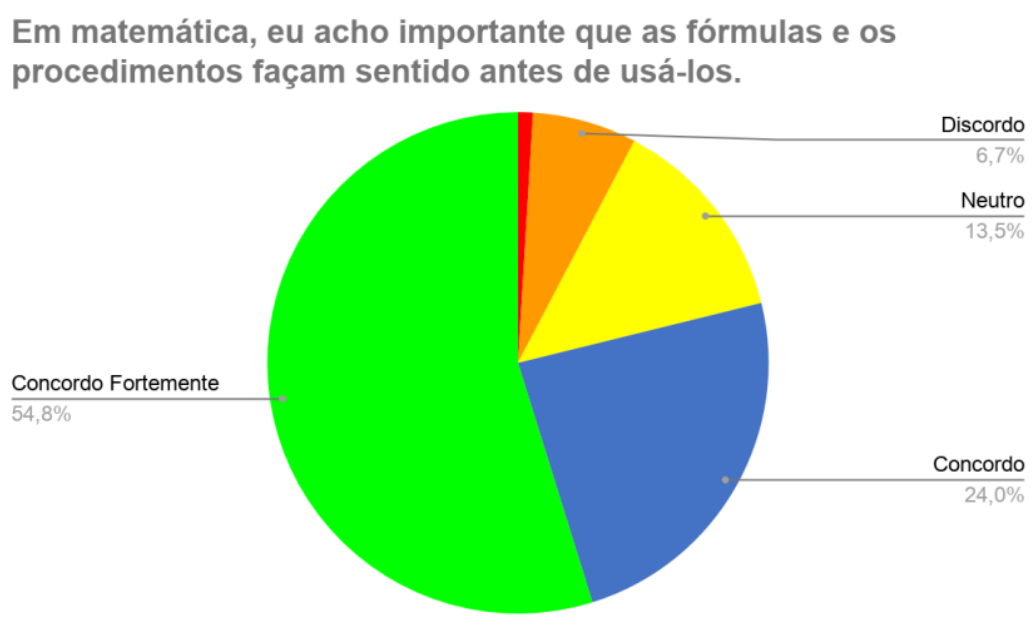

\subsection{Natureza das Respostas}

Esta categoria caracteriza as opiniões dos alunos sobre a natureza das soluções para os problemas de matemática. Os alunos podem ver as respostas na matemática como certas ou erradas e as soluções que as sustentam como tendo um certo grau de rigidez. Essas visões podem afetar as concepções de matemática dos alunos e, finalmente, suas conquistas em matemática. Para exemplificar, consideremos a afirmação representativa: Eu espero que as respostas de problemas de matemática sejam números.

No Gráfico 8 percebe-se que a dificuldade de generalização por parte dos alunos, uma habilidade essencial na aprendizagem matemática, é uma realidade. 44,3\% dos entrevistados concordaram com a sentença enquanto $29,8 \%$ dos alunos discordaram da afirmação.

\section{Gráfico 8 - Resultados para a afirmação representativa da categoria Respostas.}

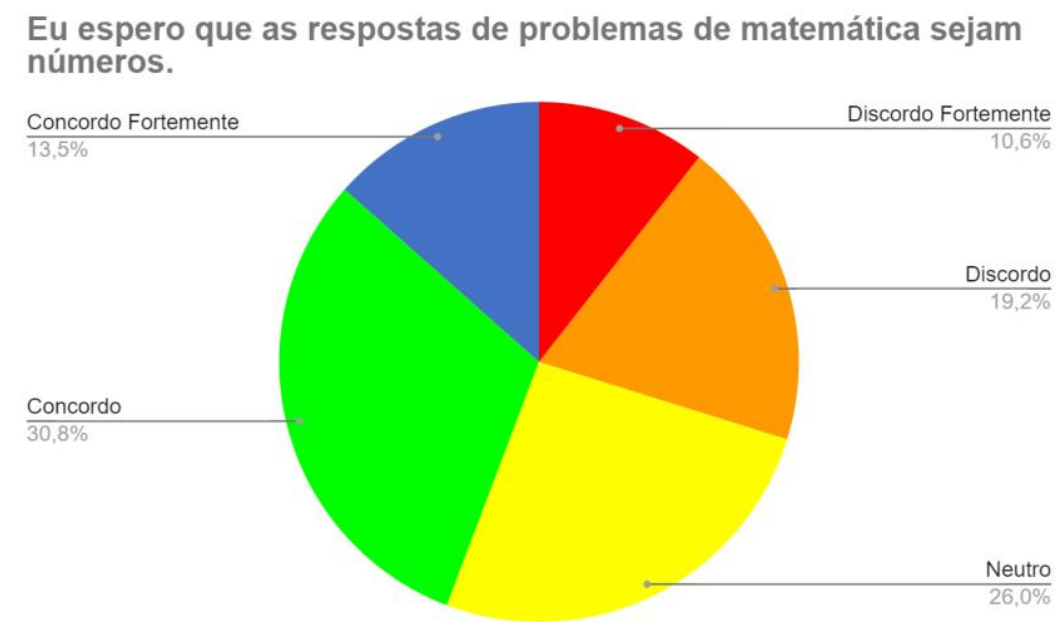

Os resultados obtidos aqui seguem, em geral, a linha dos resultados descritos por Code et al. (2016) para alunos do segundo ano de graduação, apesar de países e contextos socioeconômicos distintos. No estudo citado, as categorias Coerência e Relação com o mundo real também apresentam as melhores correspondências ao passo que as categorias Persistência e Confiança apresentaram menores índices de consonância. A diferença mais significativa entre os dois trabalhos foi encontrada na categoria natureza das respostas, que apresentou alto índice de concordância no trabalho de Code et al (2016) - em torno de 70\% de 
respostas em concordância com o padrão - porém em nosso estudo, apresentou baixo percentual de correspondência, em média 51,8\%.

Também é possível estabelecer uma relação entre os resultados apresentados por Marmitt et al. (2015), através de uma análise de discurso, onde os estudantes foram claros ao reconhecer que as maiores dificuldades na aprendizagem de conceitos matemáticos são a interpretação e raciocínio lógico, habilidades que em nosso trabalho estão incluídas nas categorias Maturidade e Coerência. Além disto, os alunos entrevistados lá frisaram a importância de fazer a conexão entre o que é aprendido nas disciplinas de base matemática e a sua aplicação numa tarefa ou contexto de Engenharia, assim como os nossos participantes frisaram a importância na categoria Relação da matemática com o mundo real.

\section{CONSIDERAÇÕES FINAIS}

A partir da análise dos resultados, pode-se afirmar que os alunos apresentam interesse pela matemática, no entanto, não se sentem confiantes. Juntamente com os resultados baixos apontados pela categoria Persistência, podemos associar a falta de confiança à baixa motivação e estímulo para o desenvolvimento das habilidades relacionadas à matemática.

Uma vez que o formulário foi aplicado a estudantes de engenharia, onde se supõe o contato intenso com a matemática nos semestres iniciais, alguns resultados se mostraram bastante coerentes neste sentido, como demonstração de interesse e busca por coerência e de aplicabilidade desta no cotidiano. Além disso, a escolha de uma amostra que já teria superado os primeiros períodos da graduação, condiz com as porcentagens apresentadas, em especial na categoria de Persistência, visto que, possivelmente os estudantes participantes já teriam passado por experiências desmotivantes, onde sua insistência em resolução de problemas já teria sido testada.

Por fim, podemos concluir que os estudantes de engenharia entendem a aplicação da matemática em suas vidas e se interessam pela mesma; porém, se sentem inseguros na resolução de problemas, tem dificuldades de generalizar conceitos e demostram ser pouco persistentes, adicionando mais estes obstáculos ao processo de aprendizagem. De todo modo, os alunos participantes mostraram-se bastante otimistas para desenvolver suas habilidades relacionadas a área, que, na visão deles, tem atuação bastante extensível no cotidiano.

\section{REFERÊNCIAS}

ADAMS, W.K., PERKINS, K.K., PODOLEFSKY, N.S., et al. New Instrument for Measuring Student Beliefs about Physics and Learning Physics: The Colorado Learning Attitudes About Science Survey. Physical review special topics-physics education research, v. 2, n. 1, p. 010101, 2006.

ALVES, M.; COUTINHO, C.; ROCHA, A. M.; RODRIGUES, C. Fatores que influenciam a aprendizagem de conceitos matemáticos em cursos de engenharia: um estudo exploratório com estudantes da Universidade do Minho. Revista Portuguesa de Educação, 29(1), pp. 259-293, 2016.

CODE, W; MERCHANT, S.; MACIEJEWSKI, W.; THOMAS, M.; LO, J. The Mathematics Attitudes and Perceptions Survey: an instrument to assess expert-like views and dispositions among undergraduate mathematics students. International Journal of Mathematical Education in Science and Technology, 47:6, p. 917-937, 2016.

FADALI, M., VELASQUEZ-BRYANT, N.; ROBINSON, M. Work in progress - Is attitude toward mathematics a major obstacle to engineering education? In: $\mathbf{3 4}^{\text {th }}$ ASEE/IEEE Frontiers in Education Conference. Savannah, GA, 2004. 
"Os desafios para formar hoje o engenheiro do amanhã"

FLANAGAN, K. M.; EINARSON, J. Gender, math confidence, and grit: Relationships with quantitative skills and performance in an undergraduate biology course. CBE-Life Sciences Education, v. 16, n. 3, p. ar47, 2017.

GYNNILD, V.; TYSSEDAL, J.; LORENTZEN, L. Approaches to study and the quality of learning: Some empirical evidence from engineering education. Int J Math Educ Sci Technol, 3, 587-607, 2005.

GOTTSCHALL, H.; GARCÍA-BAYONAS, M. Student attitudes towards group work among undergraduates in business administration, education and mathematics. Educational Research Quarterly, v. 32, n. 1, p. 3, 2008.

LESTER, F. K. Problem solving: Is it a problem. Selected issues in mathematics education, v. 36, 1980.

MARMITT, V. R.; MORAES, J. F. D.; BASSO, N. R. As atitudes e as crenças em relação à matemática: reflexos no processo de ensino e aprendizagem. Propostas interativas na educação científica e tecnológica. Porto Alegre: EDIPUCRS, 2015. 188 p.

ROCHA, L.; GUELLER, M. Fatores que permeiam o comprometimento dos alunos na aprendizagem da Matemática. In: I Congreso de Educacion Matematica de America Central y Caribe, 2013, Republica Dominicana: Santo Domingo. Anais. Santo Domingo, 2013.

SADIYAH, M., SADIJAH, C., SISWORO, HANDAYANI, F. How Students Build Their Mathematical Dispositions towards Solving Contextual and Abstract Mathematics Problems. Journal of Physics: Conference Series. Vol. 1397. No. 1. IOP Publishing, 2019.

\title{
QUANTITATIVE ANALYSIS OF ENGINEERING STUDENTS ATTITUDES IN THE MATHEMATICAL LEARNING PROCESS
}

\begin{abstract}
The perception of students about a subject can be one of the factors responsible for offering him the necessary motivation for learning. This work investigates the relationship between Mathematics and the engineering student, with the objective of measuring the importance of this relationship in the academic and personal formation of the student. For this, the MAPS (The Mathematics Attitudes and Perceptions Survey) was used as an instrument, a standardized form with 32 statements, developed at the University of British Columbia (USA) in 2016. The MAPS aim to understand the existence of a relationship between learning and a student's affinity with mathematics. These statements are separated into categories that deal with mathematical skills related to confidence, persistence, growth mindset, interest, sense making, among others. In this way, it is possible to verify in which areas the students have their perspectives closer or farther away from a pattern favorable to learning pre-established by specialists. The form was applied to a group of 104 second-and third-year students of several engineering courses at a Brazilian public institution. The results were treated statistically to correlate the responses obtained with the expected pattern for the good mathematical learning environment. The application of MAPS presented pertinent data that allowed to evaluate the existence of a link between the student's performance and its relationship of affinity with mathematics.
\end{abstract}

Keywords: Mathematical learning. Engineering. Diagnosis of attitudes. MAPS. 